\title{
Comparison of Unsatisfactory Samples from Conventional Smear versus Liquid-Based Cytology in Uterine Cervical Cancer Screening Test
}

Hoiseon Jeong · Sung Ran Hong ${ }^{1}$ Seoung-Wan Chae ${ }^{2} \cdot$ So-Young Jin ${ }^{3}$ Hye Kyoung Yoon ${ }^{4} \cdot$ Juhie Lee $^{5}$ Eun Kyung $\mathrm{Kim}^{6}$. Sook Tai $\mathrm{Ha}^{7}$ Sung Nam Kim ${ }^{8}$. Eun-Jung Park ${ }^{9}$ Jong Jae Jung ${ }^{9}$ Sun Hee Sung ${ }^{10}$ Sung-Chul Lim ${ }^{11}$

Department of Pathology, Korea University Ansan Hospital, Korea University College of Medicine, Seoul; 'Department of Pathology, Cheil General Hospital and Women's Health Care Center, Dankook University College of Medicine, Seoul; ${ }^{2}$ Department of Pathology, Kangbuk Samsung Hospital, Sungkyunkwan University School of Medicine, Seoul; ${ }^{3}$ Department of Pathology, Soonchunhyang University Hospital, Soonchunhyang University College of Medicine, Seoul; ${ }^{4}$ Department of Pathology, Inje University Busan Paik Hospital, Inje University College of Medicine, Busan; ${ }^{5}$ Department of Pathology, Kyung Hee University School of Medicine, Seoul; ${ }^{6}$ Department of Pathology, Eulji General Hospital, Seoul; ' T\&C Diagnostic Pathology Clinic, Seoul; ${ }^{8}$ Samkwang Medical Laboratories, Seoul; ${ }^{9}$ Foryou Pathology Laboratories, Gwangju; ${ }^{10}$ Department of Pathology, Ewha Womans University Mokdong Hospital, Ewha Womans University School of Medicine, Seoul; ${ }^{11}$ Department of Pathology, Chosun University School of Medicine, Gwangju, Korea

Received: December 22, 2016

Revised: March 9, 2017

Accepted: March 14, 2017

\section{Corresponding Author}

Sung-Chul Lim, MD, PhD

Department of Pathology, Chosun University School of Medicine, 309 Pilmun-daero, Dong-gu, Gwangju 61452, Korea

Tel: +82-62-230-6343

Fax: +82-62-226-5860

E-mail: sclim@chosun.ac.kr

*This study was presented at the 29th Annual Spring Meeting of the Korean Society for Cytopathologists, 2015 and the International Academy of Cytology 2016 at Yokohama.
Background: Cervical cytology for uterine cervical cancer screening has transitioned from conventional smear (CS) to liquid-based cytology (LBC), which has many advantages. The aim of this study was to compare the proportion of unsatisfactory specimens from CS versus LBC at multiple institutions including general hospitals and commercial laboratories. Methods: Each participating institution provided a minimum of 500 Papanicolaou (Pap) test results for analysis. Pap tests were classified according to the participating institution (commercial laboratory or general hospital) and the processing method (CS, ThinPrep, SurePath, or CellPrep). The causes of unsatisfactory results were classified as technical problems, scant cellularity, or complete obscuring factors. Results: A total of 38,956 Pap test results from eight general hospitals and three commercial laboratories were analyzed. The mean unsatisfactory rate of LBC was significantly lower than that of CS $(1.26 \%$ and $3.31 \%, p=.018)$. In the LBC method, samples from general hospitals had lower unsatisfactory rates than those from commercial laboratories $(0.65 \%$ vs $2.89 \%, p=.006)$. The reasons for unsatisfactory results were heterogeneous in CS. On the other hand, $66.2 \%$ of unsatisfactory results in LBC were due to the scant cellularity. Conclusions: Unsatisfactory rate of cervical cancer screening test results varies according to the institution and the processing method. LBC has a significantly lower unsatisfactory rate than $\mathrm{CS}$.

Key Words: Papanicolaou test; Cervical cytology; Unsatisfactory; Liquid-based cytology; Conventional smear 
Cervical cytology, known as Papaniolaou (Pap) test, was first introduced in Korea in the late 1950s and has since played an important role in the early detection of uterine cervical cancer. ${ }^{1}$ Conventional smear (CS) is widely used for uterine cancer screening owing to its low cost and easy application. Liquid-based cytology (LBC) was developed in the early 2000s and has recently been increasingly used in Korea. Several LBC systems are commercially available in Korea, such as ThinPrep (Cytyc Corp., Boxborough, MA, USA), BD SurePath (BD Diagnostics, Burlington, NC, USA), and CellPrep (Biodyne, Seongnam, Korea).

In LBC, the sample is prepared by rinsing the sampling tool in liquid-based medium to make a cell suspension. The cell suspension is then used to prepare a slide with an evenly-distributed monolayer sheet of cells. LBC is being marketed on the basis of its advantages, such as low unsatisfactory rates, increased sensitivity in detecting cervical precancerous lesions, standardized and automated preparations, fast screening, availability of ancillary tests including a human papillomavirus DNA test, and facilitation of computer-assisted screening. ${ }^{2}$

A number of studies have compared the performance of LBC versus $\mathrm{CS}$; however, the results have been conflicting. Some metaanalyses report that LBC has lower unsatisfactory rates and better performance in terms of sensitivity, specificity, and overall accuracy compared with CS. ${ }^{3-5}$ Other studies report no evidence that LBC reduces unsatisfactory rates or detects high-grade lesions more accurately compared with CS. ${ }^{6-8}$ An independent review also showed no significant difference between the two methods. ${ }^{9}$

Both CS and LBC are approved in Korea by the national cancer screening program as screening tests for uterine cervical cancer. However, there have been little data verifying the use of LBC in Korea, and no studies comparing the unsatisfactory rates of LBC versus that of CS in the country. To evaluate and compare the domestic use of CS and LBC, we compared the proportion of unsatisfactory tests from CS versus LBC at multiple Korean institutions including general hospitals and commercial laboratories.

\section{MATERIALS AND METHODS}

This study spanned a 6-month period from July 2014 to January 2015. Each participating institution provided a minimum of 500 Pap test results for analysis. The Pap tests were classified according to the participating institution (commercial laboratory or general hospital) and by the processing method (CS, ThinPrep, SurePath, or CellPrep). The specimens were evaluated by cytotechnologists at each given institution during their routine screening work. When an unsatisfactory result was found, the cause was evaluated using a checklist. Unsatisfactory slides were then reviewed and confirmed by cytopathologists.

The causes of unsatisfactory results were classified as either technical problems, scant cellularity, or complete obscuring factors, ${ }^{10}$ and subdivided as shown in Table 1. Technical problems referred to cases of patchy cellularity, a halo effect, and/or thick preparations in which obscuring factors such as blood, bacteria, mucus, gel, and inflammation were not present. ${ }^{10}$ Patchy cellularity referred to cases in which clear spaces formed on a slide owing to irregular loss of cells. ${ }^{10}$ Halo effect referred to cases in which a rim of cells formed on the periphery of a slide, leaving an empty space in the center. ${ }^{10}$ Thick preparation referred to cases in which the cellular details were obscured in $>75 \%$ of the slide because of aggregation of squamous epithelial cells. ${ }^{10}$ The Bethesda 2001 criteria were applied to evaluate cellularity. Regardless of the processing method, an adequate sample was defined as an estimated minimum of 5,000 well-preserved and well-visualized squamous epithelial cells. ${ }^{11}$ Cases in which $>75 \%$ of the cells were obscured were classified as unsatisfactory, ${ }^{11}$ with the exception of cases with any abnormal cells.

Statistical analysis was performed with the SPSS statistical software package ver. 16.0 (SPSS Inc., Chicago, IL, USA), by using paired $t$ tests and analysis of variance (ANOVA). Significance was defined as $\mathrm{p}<.05$.

\section{RESULTS}

We analyzed a total of 38,956 Pap test results from eight general hospitals (32,890 cases, $84.4 \%)$ and three commercial laboratories (6,066 cases, 15.6\%). Total Pap test consisted of 15,872 (general hospital, 13,299; commercial laboratory, 2,573)

Table 1. Categorization of unsatisfactory samples according to the cause

\begin{tabular}{l}
\hline Cause \\
\hline Technical problems \\
Patch cellularity \\
Halo effect \\
Thick preparation \\
Scant cellularity \\
Scant cells in a clean background \\
Scant cells in a bloody background \\
Scant cells in a background of gel/inflammation/bacteria/mucus \\
Obscuring factors \\
Blood \\
Inflammation \\
Bacteria \\
Gel \\
Mucus
\end{tabular}


cases of CS (40.7\%) and 23,084 (general hospital, 19,591; commercial laboratory, 3,493) cases of LBC $(59.3 \%)$. LBC consisted of 5,309 cases of ThinPrep (23.0\%), 15,868 cases of SurePath $(68.7 \%)$, and 1,907 cases of CellPrep (8.3\%) (Table 2). Each institution used CS in parallel with one type of LBC, with the exception of one general hospital that did not provide any CS data. The participating institutions provided data on the number of unsatisfactory samples and the total numbers of CS and LBC samples in the 6-month period. Unsatisfactory rates were calculated from the data and are shown in Table 3. The unsatisfactory rate of CS had a wide range of distribution from $0.1 \%$ to $11.2 \%$, whereas that of LBC was between $0.0 \%$ and $4.4 \%$. The mean unsatisfactory rates for CS and LBC were $3.31 \%$ and $1.26 \%$, respectively (Table 4 ), showing that LBC had a significantly lower unsatisfactory rate than CS ( $\mathrm{p}=.017)$.

The unsatisfactory rates were then analyzed according to the participating institution: either commercial laboratory or general hospital (Table 4). In commercial laboratories, the unsatisfactory rates of CS ranged from $2.91 \%$ to $11.16 \%$ (mean, $6.04 \%$ ), whereas those of LBC ranged from $1.63 \%$ to $4.37 \%$ (mean, $2.89 \%$ ). In general hospitals, the unsatisfactory rates of CS ranged

Table 2. Composition of the samples

\begin{tabular}{lccc}
\hline Method & $\begin{array}{c}\text { General } \\
\text { hospital }\end{array}$ & $\begin{array}{c}\text { Commercial } \\
\text { laboratory }\end{array}$ & Total \\
\hline Liquid-based cytology & 19,591 & 3,493 & $23,084(59.3)$ \\
Conventional smear & 13,299 & 2,573 & $15,872(40.7)$ \\
Total & $32,890(84.4)$ & $6,066(15.6)$ & $38,856(100)$ \\
\hline
\end{tabular}

Values are presented as number (\%). from $0.14 \%$ to $5.20 \%$ (mean, $2.14 \%$ ), whereas those of LBC ranged from $0 \%$ to $1.80 \%$ (mean, $0.65 \%$ ). Regardless of the processing method, the general hospital group had a significantly lower unsatisfactory rate than the commercial laboratory group $(\mathrm{p}=.006)$.

For the preparation of LBC, six institutions used ThinPrep, three used SurePath, and two used CellPrep. The unsatisfactory rates were analyzed according to the three LBC products used. The unsatisfactory rate for ThinPrep, which was used in most institutions, ranged from $0.1 \%$ to $4.4 \%$, with a mean rate of $1.6 \%$. The unsatisfactory rate for SurePath ranged from $0.0 \%$ to $0.8 \%$, with a mean rate of $0.3 \%$. Between the two institutions that used CellPrep, one reported an unsatisfactory rate of $1.6 \%$ whereas the other reported $1.8 \%$; the mean unsatisfactory rate of CellPrep was $1.7 \%$. Although SurePath $(0.3 \%)$ had a lower mean unsatisfactory rate than ThinPrep (1.6\%) or CellPrep (1.7\%), ANOVA revealed no statistically significant difference among the three groups.

The causes of unsatisfactory results were heterogeneous in CS (Table 5). The most common reason was scant cellularity on a clean background, which was observed in 90 out of 287 unsatisfactory cases of CS (31.4\%). The second most common reason was obscuring due to inflammation (84 cases, 29.3\%). A considerable proportion of unsatisfactory cases showed scant cellularity on a background of gel/inflammation/blood/mucus (GIBM; 32 cases). Inflammation was the most common obscuring factor, occurring in 84 cases; while the other factors (blood, bacteria, gel, and mucus) occurred in a minority of cases. Within the cat-

Table 3. Unsatisfactory rate (\%) of each participating institution

\begin{tabular}{|c|c|c|c|c|c|c|c|c|c|c|c|c|}
\hline \multirow{3}{*}{ Method } & \multicolumn{11}{|c|}{ Institution No. ${ }^{a}$} & \multirow{3}{*}{ Total } \\
\hline & 1 & 2 & 3 & 4 & 5 & 6 & 7 & 8 & 9 & 10 & 11 & \\
\hline & SurePath & SurePath & CellPrep & ThinPrep & ThinPrep & ThinPrep & SurePath & ThinPrep & ThinPrep & ThinPrep & CellPrep & \\
\hline LBC & $\begin{array}{c}0 / 500 \\
(0.0)\end{array}$ & $\begin{array}{c}22 / 2,856 \\
(0.8)\end{array}$ & $\begin{array}{c}9 / 500 \\
(1.8)\end{array}$ & $\begin{array}{c}9 / 539 \\
(1.7)\end{array}$ & $\begin{array}{c}1 / 1,457 \\
(0.1)\end{array}$ & $\begin{array}{c}1 / 384 \\
(0.3)\end{array}$ & $\begin{array}{c}1 / 12,512 \\
(0.0)\end{array}$ & $\begin{array}{c}5 / 843 \\
(0.6)\end{array}$ & $\begin{array}{c}55 / 1,258 \\
(4.4)\end{array}$ & $\begin{array}{c}22 / 828 \\
(2.7)\end{array}$ & $\begin{array}{c}23 / 1,407 \\
(1.6)\end{array}$ & $\begin{array}{c}148 / 23,084 \\
(0.6)\end{array}$ \\
\hline CS & $\begin{array}{c}4 / 531 \\
(0.8)\end{array}$ & $\begin{array}{c}51 / 1,155 \\
(4.4)\end{array}$ & $\begin{array}{c}26 / 500 \\
(5.2)\end{array}$ & $\begin{array}{c}23 / 658 \\
(3.5)\end{array}$ & $\begin{array}{c}19 / 2,975 \\
(0.6)\end{array}$ & $\begin{array}{c}16 / 4,670 \\
(0.3)\end{array}$ & $\begin{array}{c}4 / 2,810 \\
(0.1)\end{array}$ & No data & $\begin{array}{c}77 / 690 \\
(11.2)\end{array}$ & $\begin{array}{c}24 / 826 \\
(2.9)\end{array}$ & $\begin{array}{c}43 / 1,057 \\
(4.1)\end{array}$ & $\begin{array}{c}287 / 15,872 \\
(1.8)\end{array}$ \\
\hline
\end{tabular}

Values are presented as number (\%).

LBC, liquid-based cytology; CS, conventional smear.

${ }^{a} 1$ to 8 , general hospitals; 9 to 11 , commercial laboratories.

Table 4. Comparison of unsatisfactory sample rate according to the processing method and institution

\begin{tabular}{|c|c|c|c|c|c|}
\hline \multirow{3}{*}{ Method } & \multicolumn{5}{|c|}{ Unsatisfactory sample rate } \\
\hline & & \multirow{2}{*}{$p$-value } & \multicolumn{2}{|c|}{ Institution } & \multirow{2}{*}{$p$-value } \\
\hline & & & $\mathrm{GH}$ & $\mathrm{CL}$ & \\
\hline LBC & $1.26 \pm 1.36$ & .017 & $0.65 \pm 0.73$ & $2.89 \pm 1.38$ & .006 \\
\hline CS & $3.31 \pm 3.33$ & & $2.14 \pm 2.15$ & $6.04 \pm 4.47$ & .088 \\
\hline
\end{tabular}

Values are presented as mean \pm standard deviation.

GH, general hospital; CL, commercial laboratory; LBC, liquid-based cytology; CS, conventional smear. 
Table 5. Comparison of unsatisfactory samples in CS versus LBC

\begin{tabular}{lccc}
\hline Category & CS & LBC & p-value \\
\hline Technical problems & & & \\
$\quad$ Patchy & $13(4.5)$ & $4(2.7)$ & .06 \\
Halo & $3(1.1)$ & $18(12.2)$ & .14 \\
Thick & $24(8.4)$ & $2(1.4)$ & .17 \\
Scant cellularity & & & \\
Clean & $90(31.4)$ & $69(46.6)$ & .39 \\
Bloody & $13(4.5)$ & $22(14.9)$ & .15 \\
GlBM & $32(11.2)$ & $7(4.7)$ & .32 \\
Obscuring factors & & & \\
Blood & $15(5.2)$ & $10(6.8)$ & .44 \\
Inflammation & $84(29.3)$ & $15(10.1)$ & .09 \\
Bacteria & $5(1.7)$ & $1(0.7)$ & - \\
Gel & $2(0.7)$ & 0 & - \\
Mucus & $6(2.1)$ & 0 & - \\
Total & $287(100)$ & $148(100)$ & \\
\hline
\end{tabular}

Values are presented as number (\%).

CS, conventional smear; LBC, liquid-based cytology.

${ }^{a}$ Scant cells in a background of gel/inflammation/bacteria/mucus.

egory of technical problems, unsatisfactory cases due to a thick preparation had the largest proportion (24 cases).

Among 148 unsatisfactory LBC cases, 98 (66.2\%) were due to scant cellularity. Of note, all of the unsatisfactory cases with SurePath were due to scant cellularity. Of the remaining unsatisfactory LBC cases, 26 (17.6\%) were due to obscuring factors and $24(16.3 \%)$ were due to technical problems. Within the scant cellularity category, most cases (69 cases, 46.6\%) were on a clean background, analogous to CS cases. However, only seven LBC cases $(4.7 \%)$ showed scant cellularity on a GIBM background, whereas a considerable proportion of CS cases with scant cellularity had a GIBM background. The number and proportion of unsatisfactory LBC cases with obscuring factors (26 cases, $17.6 \%$ ) were much smaller than those of CS cases (112 cases, 39\%). There was no unsatisfactory LBC case due to obscuring by gel or mucus. Obscuring inflammation, which was the second most common cause of unsatisfactory results in CS (84 cases, 29.3\%), was much less prevalent in LBC cases (15 cases, $10.1 \%$ ). Within the technical problem category, 18 out of 24 cases were due to a halo effect, 17 of which were reported in ThinPrep cases.

There was no statistically significant difference in the unsatisfactory rate according to processing method (CS vs LBC) (Table 5). The causes of unsatisfactory results in CS cases were thick preparation, scant cellularity on a clean or GIBM background, and obscuring inflammation. The causes of unsatisfactory results in LBC samples were scant cellularity on a clean or bloody background, halo effect, and obscuring inflammation. Of note, no LBC samples were reported as unsatisfactory due to obscuring by gel or mucus; however, we were unable to verify this statistically.

\section{DISCUSSION}

Estimating the unsatisfactory rate and overall accuracy of uterine cervical cancer screening tests can help in determining the optimal screening frequency, a management plan for abnormal results, and the need to apply new methods or conduct further studies. ${ }^{5}$ A low unsatisfactory rate can decrease the chance of patient revisit, thereby lowering the overall cost of the screening program. ${ }^{12}$ Studies have shown that a substantial fraction of falsenegative Pap test results are in fact unsatisfactory results, ${ }^{13,14}$ and false-negative results can lead to worse conditions than do false-positive results. Therefore, a low unsatisfactory rate is considered an important checkpoint before adopting a particular testing protocol into a large-scale screening program. In countries with nationalized health-care systems, such as England, cervical cancer screening programs have switched from CS to LBC methods, and a striking subsequent reduction in the proportion of unsatisfactory slides has been reported., ${ }^{912}$ The Korean guideline for cervical cancer screening was established by the National Cervical Cancer Screening Guideline Development Committee and published in 2015. ${ }^{15}$ One of the key questions forwarded by the committee was regarding the accuracy and insufficient sample rate of LBC compared with CS. ${ }^{15}$ The results of the present study show that the unsatisfactory rate is significantly lower for LBC than for CS, in line with the results of previous studies. ${ }^{3,16-19}$ Considering that the performance of LBC was not inferior to that of CS in published papers from Korea, the lower unsatisfactory rate of LBC makes it a better screening test tool than CS. The current published Korean guideline for cervical cancer screening allows the use of both CS and LBC as screening tests. ${ }^{15}$

In Korea, $>60 \%$ of cervical cancer screening tests conducted by primary health-care units are analyzed in commercial laboratories. ${ }^{1,20}$ However, in most of the existing literature comparing CS and LBC, the specimens were analyzed in university hospitals. ${ }^{5}$ Thus, the present study included three commercial laboratories among the 11 participating institutions. LBC had significantly lower unsatisfactory rates in the general hospital group than in the commercial laboratory group. In the case of CS, the general hospital group had lower unsatisfactory rates than the commercial laboratory group, although the difference was not statistically significant. Considering that many parts of the sample-processing procedure are standardized for LBC, the difference in unsatisfactory rate between commercial laboratories and general hospitals is believed to be due to differences in patient 
characteristics. In general hospitals, patients who have been diagnosed as having atypical squamous cells of undetermined significance or higher cytologic atypia at their first screening are often referred to pathologists and reexamined. A precondition exists that there must be no atypical cells in a sample judged to be unsatisfactory; thus, the high fraction of patients with cytologic atypia likely contributes to the lower unsatisfactory rate in general hospitals than in commercial laboratories.

In terms of the particular LBC product used, six of the 11 participating institutions used ThinPrep, three used SurePath, and two used CellPrep. There was no significant difference in unsatisfactory rates for tests conducted using the three different LBC products, although SurePath tended to have a lower unsatisfactory rate compared to ThinPrep and CellPrep, which were similar to one another. These results are in line with a previous study showing that SurePath has a lower unsatisfactory rate than ThinPrep. ${ }^{21,22}$ Of note, one confounding variable could be that all three institutions using SurePath were general hospitals.

The analysis revealed that in CS, unsatisfactory test results were caused by a variety of factors, whereas in LBC, two-thirds of unsatisfactory samples were caused by scant cellularity. This is consistent with a previous study concluding that LBC eliminates most sources of unsatisfactory tests with the exception of scant cellularity, which is the main reason for unsatisfactory LBC tests. ${ }^{23}$ In particular, obscuring factors, which were the cause of a large proportion of the unsatisfactory CS test results, were found to be less frequent in the LBC method. It is noteworthy that we found no unsatisfactory LBC cases caused by gel or mucus obscuration. With regard to the technical problems, many unsatisfactory CS samples were caused by a thick smear, whereas those in LBC were caused by a halo effect. Of note, 17 of the 18 unsatisfactory samples caused by a halo effect were reported by institutions that used ThinPrep; the halo effect of ThinPrep is confirmed by another study. ${ }^{24}$ However, there were no significant differences in causes of unsatisfactory results between CS and LBC.

In this study, we did not use the split-sample method in which a given sample is divided and processed using both CS and LBC to compare results. The processing method was chosen at the discretion of the patient and clinician, who were unaware of the study. We assumed that random assignment was made, though there may indeed have been selection bias. Furthermore, the study was conducted with data from various institutions, and although we provided guidance on determining unsatisfactory results and their causes, the possibility of interobserver variability cannot be ruled out. The lack of epidemiological data is also a limitation of this study. In a multicenter study analyzing the effects of transitioning from CS to LBC on unsatisfactory Pap test results, an increased relative risk of unsatisfactory result was reported in women aged 50 years or older after transition to $\mathrm{LBC}{ }^{25} \mathrm{~A}$ study of randomized clinical trials in Europe reported that there is a greater decrease in unsatisfactory results with $\mathrm{LBC}$ than with CS in younger women, and that this effect decreases with age. ${ }^{19}$ However, in young women with obscuring elements, LBC seemed to have little or no effect on cervical atrophy and the consequent low cellularity problem. ${ }^{25}$ Furthermore, in comparison with the large number of cervical cancer screening tests analyzed in commercial laboratories, our commercial laboratory group was small. Therefore, there is a limitation in that the results of this study may not accurately reflect the situation in Korea. Given that the commercial laboratory group tended to show a higher rate of unsatisfactory results than the general hospital group, the overall unsatisfactory rate of cervical cancer screening test may be higher than that presented in this study.

In summary, the unsatisfactory rate of uterine cervical Pap smear results was lower for LBC than for CS. Regardless of the processing method, lower rates of unsatisfactory results were observed in the general hospital group than in the commercial laboratory group. Furthermore, we confirmed the effect of using each of the three most common LBC products. Although SurePath tended to show a lower unsatisfactory rate than did ThinPrep or CellPrep, there was no statistically significant difference. The causes of the unsatisfactory results were heterogeneous in CS, whereas most (two-third) of the unsatisfactory LBC were due to scant cellularity.

We compared the unsatisfactory rates of CS and LBC for uterine cervical cancer screening tests in Korea through a crosssectional study of approximately 39,000 Pap test results obtained during a 6-month period from 11 different institutions including both general hospitals and commercial laboratories. Our results suggest that LBC is the preferred method in Korea, and this was verified by comparing the unsatisfactory rates of Pap test results. This study provides the basic data necessary for evidence-based decision making in health-care policies such as the national cancer screening project.

\section{Conflicts of Interest}

No potential conflict of interest relevant to this article was reported. 


\section{Acknowledgments}

This research was supported by The Korean Society for Cytopathologists Grant No. 2014-01.

\section{REFERENCES}

1. Park MH. Cervical cancer screening in Korea. Korean J Cytopathol 2003; 14: 43-52.

2. Hoda RS, Loukeris K, Abdul-Karim FW. Gynecologic cytology on conventional and liquid-based preparations: a comprehensive review of similarities and differences. Diagn Cytopathol 2013; 41: 257-78.

3. Bernstein SJ, Sanchez-Ramos L, Ndubisi B. Liquid-based cervical cytologic smear study and conventional Papanicolaou smears: a metaanalysis of prospective studies comparing cytologic diagnosis and sample adequacy. Am J Obstet Gynecol 2001; 185: 308-17.

4. Abulafia O, Pezzullo JC, Sherer DM. Performance of ThinPrep liquid-based cervical cytology in comparison with conventionally prepared Papanicolaou smears: a quantitative survey. Gynecol Oncol 2003; 90: 137-44.

5. Oh JK, Shin HR, Gong G, Sohn JH, Khang SK. Diagnostic accuracy of conventional Pap test, liquid-based cytology and human papillomavirus DNA testing in cervical cancer screening in Korea: a meta-analysis. Korean J Epidemiol 2008; 30: 178-87.

6. Kituncharoen S, Tantbirojn P, Niruthisard S. Comparison of unsatisfactory rates and detection of abnormal cervical cytology between conventional Papanicolaou smear and liquid-based cytology (Sure Path(R)). Asian Pac J Cancer Prev 2015; 16: 8491-4.

7. Davey E, Barratt A, Irwig L, et al. Effect of study design and quality on unsatisfactory rates, cytology classifications, and accuracy in liquid-based versus conventional cervical cytology: a systematic review. Lancet 2006; 367: 122-32.

8. Siebers AG, Klinkhamer PJ, Grefte JM, et al. Comparison of liquidbased cytology with conventional cytology for detection of cervical cancer precursors: a randomized controlled trial. JAMA 2009; 302: 1757-64.

9. Obwegeser J, Schneider V. Thin-layer cervical cytology: a new metaanalysis. Lancet 2006; 367: 88-9.

10. Rosa M, Pragasam P, Saremian J, Aoalin A, Graf W, Mohammadi A. The unsatisfactory ThinPrep Pap test: analysis of technical aspects, most common causes, and recommendations for improvement. Diagn Cytopathol 2013; 41: 588-94.

11. Solomon D, Nayar R. The Bethesda system for reporting cervical cytology: definitions, criteria, and explanatory notes. 2nd ed. New
York: Springer, 2004.

12. Biro C, Hyne S, Roberts J, Thurloe J, Bowditch R. Liquid-based versus conventional cervical cytology. Lancet 2006; 367: 1481-2.

13. Pairwuti S. False-negative Papanicolaou smears from women with cancerous and precancerous lesions of the uterine cervix. Acta Cytol 1991; 35: 40-6.

14. van der Graaf Y, Vooijs GP, Gaillard HL, Go DM. Screening errors in cervical cytologic screening. Acta Cytol 1987; 31: 434-8.

15. Min KJ, Lee YJ, Suh M, et al. The Korean guideline for cervical cancer screening. J Gynecol Oncol 2015; 26: 232-9.

16. Ronco G, Cuzick J, Pierotti P, et al. Accuracy of liquid based versus conventional cytology: overall results of new technologies for cervical cancer screening: randomised controlled trial. BMJ 2007; 335: 28.

17. Guidos BJ, Selvaggi SM. Use of the Thin Prep Pap test in clinical practice. Diagn Cytopathol 1999; 20: 70-3.

18. Strander B, Andersson-Ellström A, Milsom I, Rådberg T, Ryd W. Liquid-based cytology versus conventional Papanicolaou smear in an organized screening program: a prospective randomized study. Cancer 2007; 111: 285-91.

19. Castle PE, Bulten J, Confortini M, et al. Age-specific patterns of unsatisfactory results for conventional Pap smears and liquid-based cytology: data from two randomised clinical trials. BJOG 2010; 117: 1067-73.

20. Lee HK, Kim SN, Khang SK, Kang CS, Yoon HK. Quality control program and its results of Korean Society for Cytopathologists. Korean J Cytopathol 2008; 19: 65-71.

21. Moriarty AT, Clayton AC, Zaleski S, et al. Unsatisfactory reporting rates: 2006 practices of participants in the college of american pathologists interlaboratory comparison program in gynecologic cytology. Arch Pathol Lab Med 2009; 133: 1912-6.

22. Fontaine D, Narine N, Naugler C. Unsatisfactory rates vary between cervical cytology samples prepared using ThinPrep and SurePath platforms: a review and meta-analysis. BMJ Open 2012; 2: e000847.

23. Siebers AG, Klinkhamer PJ, Vedder JE, Arbyn M, Bulten J. Causes and relevance of unsatisfactory and satisfactory but limited smears of liquid-based compared with conventional cervical cytology. Arch Pathol Lab Med 2012; 136: 76-83.

24. Song LH, Goh ES, Phang LC, Poh WT, Tay SK. Technical aspect of ThinPrep. Singapore Med J 2000; 41: 575-8.

25. Owens CL, Peterson D, Kamineni A, et al. Effects of transitioning from conventional methods to liquid-based methods on unsatisfactory Papanicolaou tests: results from a multicenter US study. Cancer Cytopathol 2013; 121: 568-75. 